\title{
Spherical Solution of Classical Quantum Gravity
}

\author{
Sangwha-Yi* \\ Department of Math, Taejon University, Saudi Arabia.
}

*Corresponding Author: Sangwha-Yi, Department of Math, Taejon University, Saudi Arabia.

\begin{abstract}
In the general relativity theory, using Einstein's gravity field equation, we discover the spherical solution of the classical quantum gravity. The careful point is that this theory is different from the other quantum theory. This theory is made by the Einstein's classical field equation.
\end{abstract}

Keywords: General relativity theory, Classical quantum gravity, Spherical quantum solution

PACS: $04,04.90 .+e, 04.60$

\section{INTRODUCTION}

This theory's aim is that we discover the spherical solution of the quantum gravity.

We can think the following formula.

$$
\alpha=\frac{h c}{G M^{2}} \text { is non-Dimension number. } \alpha \text { 's Dimension is } \frac{\mathrm{J} \cdot \mathrm{s} \cdot \mathrm{m} / \mathrm{s}}{\mathrm{N} \cdot \mathrm{m}^{2} \cdot \mathrm{kg}^{2} / \mathrm{kg}}=\frac{\mathrm{J} \cdot \mathrm{m}}{\mathrm{J} \cdot \mathrm{m}}=1
$$

$h$ is the plank constant, $c$ is the light speed, $G$ is the gravity constant, $M$ is the matter's mass.

The classical vacuum solution (Schwarzschild solution) of the general relativity is

$$
d \tau^{2}=\left(1-\frac{2 G M}{r c^{2}}\right) d t^{2}-\frac{1}{c^{2}}\left[\frac{d r^{2}}{1-\frac{2 G M}{r c^{2}}}+r^{2} d \theta^{2}+r^{2} \sin ^{2} \theta d \phi^{2}\right]
$$

\section{SPHERICAL QUANTUM SOlUTION In VACUUM STATE}

In this theory, the general relativity theory's field equation is written completely.

$$
R_{\mu \nu}-\frac{1}{2} g_{\mu v} R=-\frac{8 \pi G}{c^{4}} T_{\mu \nu}
$$

The Ricci tensor is by $T_{\mu \nu}=0$ in vacuum state.

$$
R_{\mu v}=0
$$

The proper time of spherical coordinates is

$$
d \tau^{2}=A(t, r) d t^{2}-\frac{1}{c^{2}}\left[B(t, r) d r^{2}+r^{2} d \theta^{2}+r^{2} \sin ^{2} \theta d \phi^{2}\right]
$$

If we use $\mathrm{Eq}(5)$, we obtain the Ricci-tensor equations.

$$
\begin{aligned}
& R_{t t}=-\frac{A^{\prime \prime}}{2 B}+\frac{A^{\prime} B^{\prime}}{4 B^{2}}-\frac{A^{\prime}}{B r}+\frac{A^{\prime 2}}{4 A B}+\frac{\ddot{B}}{2 B}-\frac{\dot{B}^{2}}{4 B^{2}}-\frac{\dot{A} \dot{B}}{4 A B}=0 \\
& R_{r r}=\frac{A^{\prime \prime}}{2 A}-\frac{A^{\prime 2}}{4 A^{2}}-\frac{A^{\prime} B^{\prime}}{4 A B}-\frac{B^{\prime}}{B r}-\frac{\ddot{B}}{2 A}+\frac{\dot{A} \dot{B}}{4 A^{2}}+\frac{\dot{B}^{2}}{4 A B}=0 \\
& R_{\theta \theta}=-1+\frac{1}{B}-\frac{r B^{\prime}}{2 B^{2}}+\frac{r A^{\prime}}{2 A B}=0
\end{aligned}
$$


$R_{\phi \phi}=R_{\theta \theta} \sin ^{2} \theta=0$

$R_{t r}=-\frac{\dot{B}}{B r}=0$

$R_{t \theta}=R_{t \phi}=R_{r \theta}=R_{r \phi}=R_{\theta \phi}=0$

In this time, $\quad=\frac{\partial}{\partial r}, \cdot=\frac{1}{c} \frac{\partial}{\partial t}$

By $\operatorname{Eq}(10)$,

$\dot{B}=0$

By $\operatorname{Eq}(6)$ and $\operatorname{Eq}(7)$,

$$
\frac{R_{t t}}{A}+\frac{R_{r r}}{B}=-\frac{1}{B r}\left(\frac{A^{\prime}}{A}+\frac{B^{\prime}}{B}\right)=-\frac{(A B)^{\prime}}{r A B^{2}}=0
$$

Hence, we obtain this result.

$$
A=\frac{1}{B}
$$

If $\mathrm{Eq}(14)$ inserts $\mathrm{Eq}(8)$,

$$
R_{\theta \theta}=-1+\frac{1}{B}-\frac{r B^{\prime}}{2 B^{2}}+\frac{r A^{\prime}}{2 A B}=-1+\left(\frac{r}{B}\right)^{\prime}=0
$$

If we solve $\operatorname{Eq}(15)$,

$$
\frac{r}{B}=r+C \rightarrow \frac{1}{B}=1+\frac{C}{r}
$$

In this time, we are able to think the following formula.

$$
\begin{aligned}
& C=-\frac{2 G M}{c^{2}} \exp \left[-\alpha_{1}\left(\frac{h c}{G M^{2}}\right)^{\beta_{1}}-\alpha_{2}\left(\frac{h c}{G M^{2}}\right)^{\beta_{2}}-\ldots \alpha_{i}\left(\frac{h c}{G M^{2}}\right)^{\beta_{i}} \ldots-\alpha_{n}\left(\frac{h c}{G M^{2}}\right)^{\beta_{n}}\right] \\
& N_{i}>\alpha_{i} \geq 0, \beta_{i} \geq 1-\varepsilon_{0 i}, 0<\varepsilon_{0 i}<<1 \\
& \alpha_{1}, \alpha_{2}, \ldots \alpha_{n}, \beta_{1}, \beta_{2}, \ldots \beta_{n} \text { are real numbers. }
\end{aligned}
$$

$N_{i}$ is the large number. $\varepsilon_{0 i}$ is the smallest number.

The reason of $\beta_{i} \geq 1-\varepsilon_{0 i}$ is because if $0 \leq \beta_{i}<1-\varepsilon_{0 i}$, we are not able to represent the real gravity situation. In this time, the large number $N_{i}$ is the number that befit the real gravity situation.

The smallest number $\varepsilon_{0 i}$ is the positive number.

Therefore, $\mathrm{Eq}(14)$ is

$$
\begin{aligned}
& A=\frac{1}{B}=1-\frac{2 G M}{r c^{2}} \sum(M) \\
& \sum(M)=\exp \left[-\alpha_{1}\left(\frac{h c}{G M^{2}}\right)^{\beta_{1}}-\alpha_{2}\left(\frac{h c}{G M^{2}}\right)^{\beta_{2}}-\ldots \alpha_{i}\left(\frac{h c}{G M^{2}}\right)^{\beta_{i}}-\ldots-\alpha_{n}\left(\frac{h c}{G M^{2}}\right)^{\beta_{n}}\right] \\
& N_{i}>\alpha_{i} \geq 0, \beta_{i} \geq 1-\varepsilon_{0 i}, 0<\varepsilon_{0 i}<<1 \\
& \alpha_{1}, \alpha_{2}, \ldots \alpha_{n}, \beta_{1}, \beta_{2}, \ldots \beta_{n} \text { are real numbers. } \\
& N_{i} \text { is the large number. } \varepsilon_{0 i} \text { is the smallest number. }
\end{aligned}
$$


If we want to know the gravity acceleration of Newton's limitation,

$$
\frac{d^{2} r}{d t^{2}} \approx \frac{1}{2} c^{2} \frac{\partial(-A)}{\partial r}=-\frac{G M}{r^{2}} \exp \left[-\alpha_{1}\left(\frac{h c}{G M^{2}}\right)^{\beta_{1}}-\alpha_{2}\left(\frac{h c}{G M^{2}}\right)^{\beta_{2}}-\ldots-\alpha_{n}\left(\frac{h c}{G M^{2}}\right)^{\beta_{n}}\right]
$$

Therefore, the spherical solution of the quantum gravity is

$$
\begin{aligned}
& d \tau^{2}=\left(1-\frac{2 G M}{r c^{2}} \sum(M)\right) d t^{2}-\frac{1}{c^{2}}\left[\frac{d r^{2}}{\left(1-\frac{2 G M}{r c^{2}} \sum(M)\right)}+r^{2} d \theta^{2}+r^{2} \sin ^{2} \theta d \phi^{2}\right] \\
& \sum(M)=\exp \left[-\alpha_{1}\left(\frac{h c}{G M^{2}}\right)^{\beta_{1}}-\alpha_{2}\left(\frac{h c}{G M^{2}}\right)^{\beta_{2}}-\ldots \alpha_{i}\left(\frac{h c}{G M^{2}}\right)^{\beta_{i}}-\ldots-\alpha_{n}\left(\frac{h c}{G M^{2}}\right)^{\beta_{n}}\right] \\
& N_{i}>\alpha_{i} \geq 0, \beta_{i} \geq 1-\varepsilon_{0 i}, 0<\varepsilon_{0 i}<<1 \\
& \alpha_{1}, \alpha_{2}, \ldots \alpha_{n}, \beta_{1}, \beta_{2}, \ldots \beta_{n} \text { are real numbers. } \\
& N_{i} \text { is the large number. } \varepsilon_{0 i} \text { is the smallest number. }
\end{aligned}
$$

\section{Classical Limitation OF SPHERICAl QuANTUM SOlution}

In $\mathrm{Eq}(20)$, if $h \rightarrow 0$,

$$
\Sigma(M)=\exp \left[-\alpha_{1}\left(\frac{h c}{G M^{2}}\right)^{\beta_{1}}-\alpha_{2}\left(\frac{h c}{G M^{2}}\right)^{\beta_{2}}-\ldots-\alpha_{n}\left(\frac{h c}{G M^{2}}\right)^{\beta_{n}}\right] \rightarrow 1
$$

In this time, $\mathrm{Eq}(20)$ does the Schwarzschild solution.

$$
d \tau^{2}=\left(1-\frac{2 G M}{r c^{2}}\right) d t^{2}-\frac{1}{c^{2}}\left[\frac{d r^{2}}{1-\frac{2 G M}{r c^{2}}}+r^{2} d \theta^{2}+r^{2} \sin ^{2} \theta d \phi^{2}\right]
$$

In $\mathrm{Eq}(20)$, if $M \rightarrow 0$,

$$
M \sum(M)=M \exp \left[-\alpha_{1}\left(\frac{h c}{G M^{2}}\right)^{\beta_{1}}-\alpha_{2}\left(\frac{h c}{G M^{2}}\right)^{\beta_{2}}-\ldots-\alpha_{n}\left(\frac{h c}{G M^{2}}\right)^{\beta_{n}}\right]=0
$$

In this time, $\mathrm{Eq}(20)$ does the Minkowski space-time.

$$
d \tau^{2}=d t^{2}-\frac{1}{c^{2}}\left[d r^{2}+r^{2} d \theta^{2}+r^{2} \sin ^{2} \theta d \phi^{2}\right]
$$

If the speed $u=0$ in gravity field, the proper time is in the classical quantum gravity solution

$$
\begin{aligned}
& u^{2}=\frac{g_{r r} d r^{2}+g_{\theta \theta} d \theta^{2}+g_{\phi \phi} d \phi^{2}}{d t^{2}}=0 \\
& d \tau^{2}=\left(1-\frac{2 G M}{r c^{2}} \sum(M)\right) d t^{2}
\end{aligned}
$$

In this time, if particles' mass are $m_{i}$, the fusion energy is $e$,

$$
E=M c^{2}=m_{1} c^{2}+m_{2} c^{2}+\ldots+m_{n} c^{2}+e
$$

In this time, if the binding energy of particle's mass $m_{i}$ and $m_{j}$ is $e_{i j}$, the proper time is

$$
\begin{aligned}
& d \tau^{2}=\left(1-\frac{2 G M}{r c^{2}} \sum(M)\right) d t^{2} \\
& =d t^{2}-\frac{2 G m_{1}}{r c^{2}} \sum\left(m_{1}\right) d t^{2}-\frac{2 G m_{2}}{r c^{2}} \sum\left(m_{2}\right) d t^{2} \ldots-\frac{2 G m_{n}}{r c^{2}} \sum\left(m_{n}\right) d t^{2}
\end{aligned}
$$


$-\left[\frac{2 G e_{12}}{r c^{4}} \sum\left(e_{12} / c^{2}\right)+\frac{2 G e_{13}}{r c^{4}} \sum\left(e_{13} / c^{2}\right)+\frac{2 G e_{23}}{r c^{4}} \sum\left(e_{23} / c^{2}\right)+\ldots+\frac{2 G e_{n(n-1)}}{r c^{4}} \sum\left(e_{n(n-1)} / c^{2}\right)\right] d t^{2}$

$\sum\left(e_{i j} / c^{2}\right)=\exp \left[-\alpha_{1}\left(\frac{h c^{5}}{G e_{i j}{ }^{2}}\right)^{\beta_{1}}-\alpha_{2}\left(\frac{h c^{5}}{G e_{i j}{ }^{2}}\right)^{\beta_{2}}-\ldots-\alpha_{n}\left(\frac{h c^{5}}{G e_{i j}{ }^{2}}\right)^{\beta_{n}}\right]$

$N_{i}>\alpha_{i} \geq 0, \beta_{i} \geq 1-\varepsilon_{0 i}, 0<\varepsilon_{0 i}<<1$

$\alpha_{1}, \alpha_{2}, \ldots \alpha_{n}, \beta_{1}, \beta_{2}, \ldots \beta_{n}$ are real numbers.

$N_{i}$ is the large number. $\varepsilon_{0 i}$ is the smallest number.

\section{CONCLUSION}

We found the spherical solution of the classical quantum gravity. Careful point is this theory is different from the other quantum theory. This theory is made by the Einstein's classical field equation.

\section{REFERENCES}

[1] S.Weinberg,Gravitation and Cosmology(John wiley \& Sons,Inc,1972)

[2] P.Bergman,Introduction to the Theory of Relativity(Dover Pub. Co.,Inc., New York,1976), Chapter V

[3] C.Misner, K,Thorne and J. Wheeler, Gravitation(W.H.Freedman \& Co.,1973)

[4] S.Hawking and G. Ellis, The Large Scale Structure of Space-Time(Cam-bridge University Press,1973)

[5] R.Adler,M.Bazin and M.Schiffer,Introduction to General Relativity(McGraw-Hill,Inc.,1965)

Citation: Sangwha-Yi, (2019). Spherical Solution of Classical Quantum Gravity. International Journal of Advanced Research in Physical Science (IJARPS) 6(8), pp.3-6, 2019.

Copyright: (C) 2019 Authors, This is an open-access article distributed under the terms of the Creative Commons Attribution License, which permits unrestricted use, distribution, and reproduction in any medium, provided the original author and source are credited. 